\title{
Myositis-myasthenia gravis overlap syndrome complicated with myasthenia crisis and myocarditis associated with anti-programmed cell death-1 (sintilimab) therapy for lung adenocarcinoma
}

\author{
Qian Xing ${ }^{1,2}$, Zhong-Wei Zhang ${ }^{1,2}$, Qiong-Hua Lin $^{1,2}$, Li-Hua Shen ${ }^{1,2}$, Peng-Mei Wang ${ }^{1,2}$, Shan Zhang $^{1,2}$, \\ Ming Fan $^{2,3}$, Biao Zhu ${ }^{1,2}$ \\ ${ }^{1}$ Department of Anaesthesia, Critical Care and Pain Medicine, Fudan University Shanghai Cancer Center, Shanghai 200032, China; ${ }^{2}$ Department of \\ Oncology, Shanghai Medical College, Fudan University, Shanghai 200032, China; ${ }^{3}$ Department of Radiation Oncology, Fudan University Shanghai \\ Cancer Center, Shanghai 200032, China \\ Correspondence to: Biao Zhu. Department of ICU, Fudan University Shanghai Cancer Center, No. 270 DongAn Road, Shanghai 200032, China. \\ Email: zhubiaozs@sohu.com.
}

\begin{abstract}
Immune checkpoint inhibitors (ICIs) have improved clinical outcomes with a number of advanced malignancies. However, diverse immune-related adverse events (iRAEs) occurred with the widespread use of ICIs, some of which are rarely and life-threatening. Here we report a 66-year-old patient with lung adenocarcinoma who received two doses of sintilimab, a human monoclonal antibody against programmed cell death-1 (PD-1), experienced a fatal storm of iRAEs. He was admitted to the intensive care unit (ICU) by immune induced-myositis/myocarditis and rhabdomyolysis. Despite immediate immunosuppressive therapy with methylprednisolone (MP) and immunoglobulin intravenously, he developed into myositis-myasthenia gravis (MG) overlap syndrome complicated with myasthenia crisis. We commenced plasma exchange (PLEX), mechanical ventilation, immunosuppressive therapy, as well as other supportive therapies. Three months later, the patient's serum creatine phosphate kinase (CPK) and anti-acetylcholine receptor antibody (anti-AChR-Ab) returned to normal despite tumor progression. Herein we discuss the incidence, operating mechanism and management strategies of the fatal iRAEs. Early admission to the ICU and multidisciplinary collaborative treatment for unstable patients with iRAEs could help to achieve a favorable outcome.
\end{abstract}

Keywords: Immune checkpoint inhibitors (ICIs); programmed cell death-1 (PD-1); immune-related adverse events (iRAEs); immune induced-myositis/myocarditis; myasthenia gravis (MG); myasthenia crisis; plasma exchange (PLEX)

Submitted Oct 23, 2019. Accepted for publication Jan 02, 2020.

doi: $10.21037 /$ atm.2020.01.79

View this article at: http://dx.doi.org/10.21037/atm.2020.01.79

\section{Introduction}

The application of immune checkpoint inhibitors (ICIs) has ushered a new era for cancer therapy and shown the efficacy of improving the survival time for patients with advanced malignancies, such as non-small cell lung cancer and metastatic melanoma $(1,2)$. ICIs potentiate $\mathrm{T}$ cells cytotoxicity against cancer cells through targeting cytotoxic T lymphocyte-associated protein-4 (CTLA-4), programmed cell death-1 (PD-1) or programmed cell death-ligand 1 (PD-L1) $(3,4)$. The blockade of PD-1 on T cells or PD-L1 on the surface of the cancer cells with antibodies enhances the anti-tumor immune activity by the $\mathrm{T}$ cells (5). However, more and more immune-related adverse events (iRAEs) have been reported with the widespread use of ICIs, some of which are severe and fatal for the patients $(6,7)$. Sintilimab $\left(\right.$ Tyvyt $\left.^{\circledR}\right)$, a human immunoglobulin G4 
(IgG4) anti-PD-1 monoclonal antibody co-developed by Innovent Biologics and Eli Lilly Company, approved for the treatment for relapsed Hodgkin's lymphoma after $\geq 2$ lines of systemic chemotherapy in China-since December 2018, is now undergoing phase I, II and III development using in various solid tumors including nonsquamous nonsmall cell lung cancer (8). Herein we present a case of lung adenocarcinoma, which developed to a fatal storm of iRAEs after two doses of sintilimab.

\section{Case presentation}

A 66-year-old man who had a smoking history of 30 years, admitted for myasthenia gravis (MG), accepted radical resection of thymoma and left lung adenocarcinoma in 2006, with postoperative pathology suggesting type $A B$ thymoma. He was found with the right lung adenocarcinoma in 2014, and underwent video-assisted thoracoscopy pulmonary wedge resection followed by three 21-day cycles of chemotherapy, which was consisted of pemetrexed $0.8 \mathrm{~g}$ and carboplatin $0.5 \mathrm{~g}$ on day 1 (PC chemotherapy).

Three years later, the left lung adenocarcinoma relapsed with the patient. After two cycles of the same PC chemotherapy followed by one course of radiotherapy to $54 \mathrm{~Gy}$ in 27 fractions, the tumor continued to progress. As immunohistochemical examination revealed high PD-L1 (test with 22C3 antibody) expression in $50 \%$ of the tumor cells from the biopsy of the left lung carcinoma, he was treated with sintilimab at a dose of $200 \mathrm{mg}$ per day intravenously at his demand out of economic reasons. And the second dose was given 21 days later. Four days after two doses of sintilimab, he experienced fatigue, myalgia and tender muscles in both the upper and lower extremities. He was hospitalized to the intensive care unit (ICU) for shortness of breath and progressive muscle weakness 8 days later. Blood results showed increased creatine phosphate kinase (CPK) up to 11,919 (normal 55-170) U/L, serum myoglobin up to over 3,000 (normal $28-72) \mathrm{ng} / \mathrm{mL}$ and troponin $\mathrm{T}$ (TnT) up to 0.916 (normal 0.013-0.025) ng/mL. Complete right bundle branch block complicated with complete atrioventricular block was seen the electrocardiogram despite normal echocardiogram, resulting in the diagnosis of an immune induced-myositis/ myocarditis and rhabdomyolysis. We immediately started an immunosuppressive therapy with methylprednisolone (MP, $2 \mathrm{mg} / \mathrm{kg} / \mathrm{d}$ ) and immunoglobulin $(400 \mathrm{mg} / \mathrm{kg} / \mathrm{d}$ for 5 days) intravenously. And a temporary pace-maker was inserted for 2 days till the normalization of heart rhythm. In the meantime, the patient presented dysphagia, hypercapnic respiratory failure, ptosis, ophthalmoplegia. He had to be put on non-invasive positive pressure ventilation (NIPPV), but eventually intubated. We diagnosed him with myositisMG overlap syndrome complicated with myasthenia crisis considering the elevated anti-acetylcholine receptor antibody (anti-AChR-Ab) to $8.97 \mathrm{nmol} / \mathrm{L}$ (positive $>0.50 \mathrm{nmol} / \mathrm{L}$ ), then adjusted the treatment with MP (500 mg daily for 5 days followed by does-reduction step by step) and pyridostigmine bromide $(120 \mathrm{mg}$, twice a day). In due time the patient was given tracheotomy, antibiotic therapy, nutrition support, ventilator weaning rehabilitation, regular diaphragm mobility assessment by ultrasound, and pulmonary hygiene. A lack of diaphragmatic activity was then confirmed. After all these treatments, symptoms in his peripheral limbs and eye opening improved, the serum level of CPK normalized and anti-AChR-Ab decreased to $2.394 \mathrm{nmol} / \mathrm{L}$ a month after admission. However, the weakness in respiratory muscles barely responded to the treatments. Since the half-life of immunoglobulin was 3-4 weeks, plasma exchange (PLEX) was not carried out until the fifth week after immunoglobulin therapy, avoiding to destroy the efficacy of immunoglobulin (9). Owing to two courses of PLEX, the patient could have normal antiAChR-Ab and breath spontaneously for 8-12 hours per day. Unfortunately, the repeat CT scan revealed the atelectasis of left upper lung from tumor progression (Figure 1), 2 months after sintilimab therapy. Three months since admission to ICU, the patient is now receiving maintenance therapy of pyridostigmine bromide without prednisone, as well as mechanical ventilation (12 hours per day), and continuing rehabilitation in the general ward. Changes in CPK, TnT and anti-AChR-Ab levels after sintilimab therapy are demonstrated in Figure 2.

\section{Discussion}

Although the incidence of high-grade iRAEs such as myocarditis induced by anti-PD-1 antibody is less than $1 \%$, death occurs from cardiac failure or arrhythmias or complications of a prolonged stay in ICU, leading to a mortality of $46 \%$ in the large series. It is reported that the patients, mostly with melanoma or lung cancers, received only one or two doses of anti-PD-1 therapy before the onset of myocarditis, and $25 \%$ of them may overlap with myositis or MG (10). Some neuromuscular iRAEs cases, especially in those with preexisting immune disease, seem more likely to suffer from overlap-syndromes 

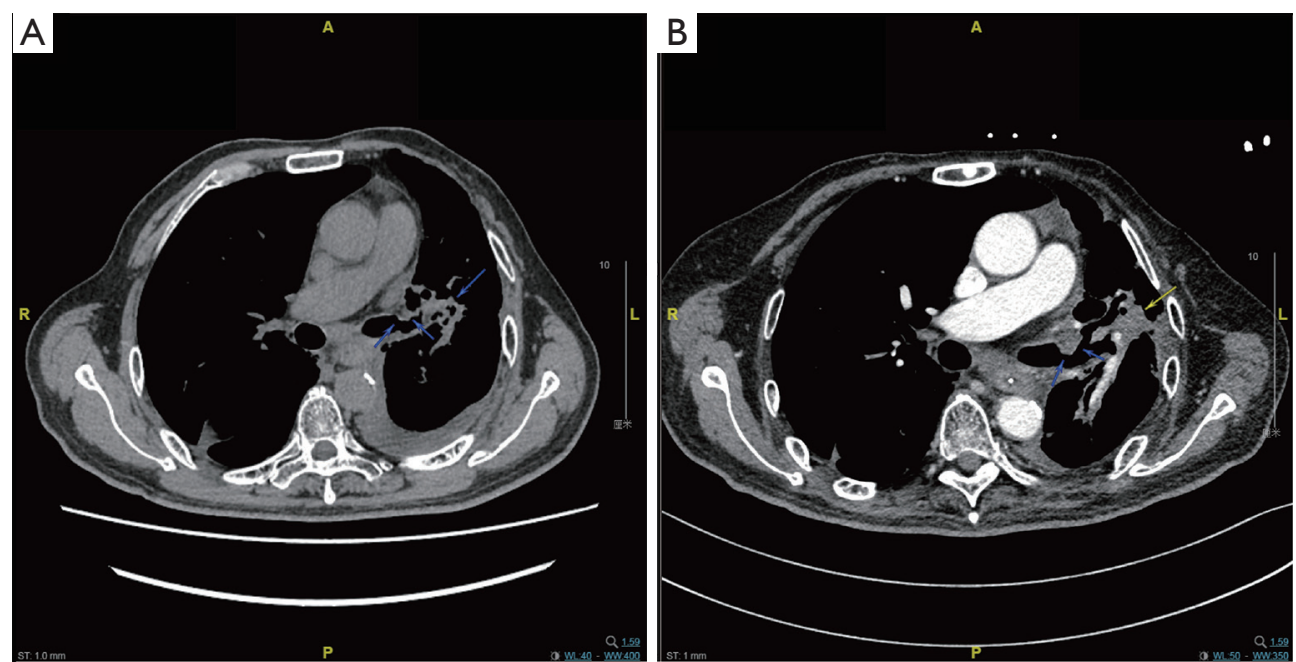

Figure 1 The comparison of CT images before and after sintilimab therapy. (A) The CT images 3 days before sintilimab therapy; (B) the CT images 2 months after sintilimab therapy. The contrast between the two images suggests the tumor continue to progress, as indicated by the arrows.

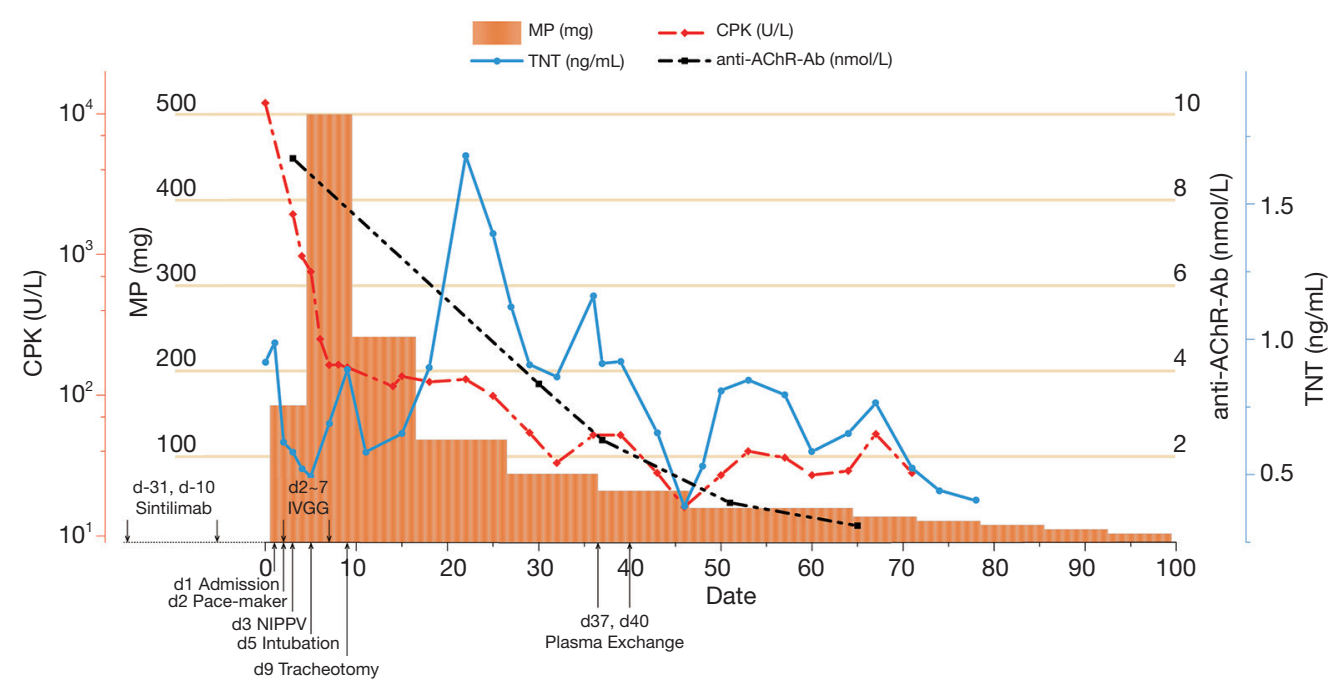

Figure 2 The clinical course after sintilimab therapy. MP, methylprednisolone; CPK, creatine phosphate kinase; TnT, troponin T; antiAChR-Ab, anti-acetylcholine receptor antibody; IVGG, intravenous gamma globulin; NIPPV, non-invasive positive pressure ventilation.

indicating myositis, myocarditis and MG present at the same time, and have higher frequencies of myasthenic crisis and fatal deterioration (10-12). The combination of checkpoint blockade is also much easier to have lifethreatening adverse effects than mono-therapy $(13,14)$. In a large retrospective study of 9,869 cancer patients in Japan with nivolumab, 12 cases $(0.12 \%)$ developed MG in 6 weeks after nivolumab and rapidly deteriorated despite immediate management, two of them died from myocarditis and myasthenic crisis. Within the-12 MG patients, 10 cases had positive anti-AChR-Ab, and 4 cases were complicated by myositis while 3 cases were accompanied by myocarditis (7). In a systematic review of 85 patients with neuromuscular disorders following anti-PD-1 therapy, more than $30 \%$ of them developed cardiac complications. Among the 23 patients diagnosed with MG from the review, 8 patients $(35 \%)$ had a history of preexisting MG while 6 of whom showing positive anti-AChR-Ab (15). In 
addition, the patient in our case did not have anti-AChR$\mathrm{Ab}$ test before sintilimab treatment. It reminded us that it would be reasonable to test for anti-AChR-Ab before the commencement of anti-PD-1 therapy to avoid the high risk of fatal iRAEs, especially in patients with thymoma or MG history. The diagnosis of immune-related MG could be based on the clinical features and the presence of serum antiAChR-Ab or anti-muscle-specific kinase antibodies (antiMuSK-Ab), however, the auto-antibodies of some patients with seronegative MG are not detectable. Electromyography could contribute to prompt diagnosis at this time $(9,16)$.

The operating mechanism of iRAEs is not clear yet. Johnson assumed that there seemed to be cross antigens between tumor tissues and striated muscle (myocardium and skeletal muscle). As a result, the distinct T-cell receptors targeted dissimilar antigens with the mislead of the clonal T-cell receptor sequences across tumor and muscle samples (17). In cases related to ICI-induced myositis/ myocarditis, the skeletal muscle and myocardium biopsy demonstrated a greater infiltration of mononuclear cells, especially CD8 $+\mathrm{T}$ cells, leading to the development of iRAEs $(7,17,18)$. Besides, muscle biopsy could act as a helpful diagnostic tool to differentiate necrotizing myopathy from MG. So, it is a pity that the patient in our case refused to take muscle biopsy for definite diagnosis. Ji et al., developed a monkey model characterized by antiPD-1 antibody-induced multiple organ toxicities including myocarditis, indicating increased proliferation in CD4+ and CD8+ T lymphocytes, as well as cardiac troponin-I and NT-pro-BNP in the myocardium (19). The study of animal models supported that either CTLA-4 or PD-1 could prevent from immune-mediated damage, for the block of CTLA4 or PD-1/PD-L1 might result in anti-tumor response and diversification of recognition to myocardium and skeletal muscle by CD8+ T cells (20).

MG has been characterized as the most frequent neuromuscular manifestations among PD-1 inhibitorassociated iRAEs, the clinicians should be aware of how to manage it $(21,22)$. The patients with ICIs-related MG or myasthenic crisis require immediate initiation of intravenous corticosteroid treatment and consideration of treatment escalation with immunoglobulins, PLEX, cyclophosphamide, rituximab, azathioprine or methotrexate $(9,11,23)$. Although immunoglobulins are proved to be equally effective with PLEX for myasthenic crisis, PLEX works more quickly and has better performance in MG patients with positive anti-MuSK-Ab $(24,25)$. Additionally, nearly half of the cases with ICIs-related myasthenic crisis require ventilatory support (7). It is really important to reduce the morbidity by comprehensive assessments of risk factors like history of heart disease, autoimmune disease and diabetes before starting the medication (20). In addition, an early prediction of iRAEs improves the outcome. An American study which enrolled 5160064 anti-PD-1 monotherapy cases from 19 different cancer types, revealed a significant positive correlation between the reporting odds ratios (RORs) of iRAE and the corresponding tumor mutational burden (TMB), which is a biomarker for prediction of therapy response. It strongly suggested cancers with a high TMB, such as melanoma and nonsmall cell lung cancer, are associated with a higher ROR of iRAE during anti-PD-1 therapy than cancers with a low TMB because of antigen spreading (26). Furthermore, physicians from both oncology and ICU department should have a good knowledge of the salient clinical features and make full use of the examinations to avoid delaying the diagnosis. And cardiac magnetic resonance imaging (MRI) scan is the gold standard noninvasive test for the diagnosis of myocarditis (27). Unfortunately, the patient in this case refused it for economic reasons. Mortality was high in the patients with high-grades iRAEs, despite immediate and adequate treatment strategies including corticosteroid, immunoglobulins, PLEX and immune-adsorption. A PD-1-induced myocarditis case suffered from malignant arrhythmia was reported to get cured by alemtuzumab on the base of fundamental anti-immunotherapy. Alemtuzumab is a monoclonal antibody that binds to CD52 of peripheral immune cells and leads to rapid cytolytic induction of immunosuppression (28). Early admission for unstable patients of iRAEs to ICU is recommended. A collaborative and multidisciplinary approach dominated by the intensivists and physicians from related departments could help to achieve a favorable outcome.

\section{Acknowledgments}

Funding: None.

\section{Footnote}

Conflicts of Interest: The authors have no conflicts of interest to declare. 
Ethical Statement: The authors are accountable for all aspects of the work in ensuring that questions related to the accuracy or integrity of any part of the work are appropriately investigated and resolved. Written informed consent was obtained from the patients for publication of this manuscript and any accompanying images.

Open Access Statement: This is an Open Access article distributed in accordance with the Creative Commons Attribution-NonCommercial-NoDerivs 4.0 International License (CC BY-NC-ND 4.0), which permits the noncommercial replication and distribution of the article with the strict proviso that no changes or edits are made and the original work is properly cited (including links to both the formal publication through the relevant DOI and the license). See: https://creativecommons.org/licenses/by-nc-nd/4.0/.

\section{References}

1. Carbone DP, Reck M, Paz-Ares L, et al. First-line nivolumab in stage IV or recurrent non-small-cell lung cancer. N Engl J Med 2017;376:2415-26.

2. Wolchok JD, Chiarion-Sileni V, Gonzalez R, et al. Overall survival with combined nivolumab and ipilimumab in advanced melanoma. N Engl J Med 2017;377:1345-56.

3. Akella P, Loganathan S, Jindal V, et al. Anti PD-1 immunotherapy related interstitial lung disease presenting as respiratory failure - a review with case series. Respir Med Case Rep 2018;26:17-22.

4. Canino C, Perrone L, Bosco E, et al. Targeting angiogenesis in metastatic renal cell carcinoma. Expert Rev Anticancer Ther 2019;19:245-57.

5. Abril-Rodriguez G, Ribas A. SnapShot: immune checkpoint inhibitors. Cancer Cell 2017;31:848-848.e1.

6. Benfaremo D, Manfredi L, Luchetti MM, et al. Musculoskeletal and rheumatic diseases induced by immune checkpoint inhibitors: a review of the literature. Curr Drug Saf 2018;13:150-64.

7. Suzuki S, Ishikawa N, Konoeda F, et al. Nivolumab-related myasthenia gravis with myositis and myocarditis in Japan. Neurology 2017;89:1127-34.

8. Hoy SM. Sintilimab: first global approval. Drugs 2019;79:341-6.

9. Sanders DB, Wolfe GI, Narayanaswami P, et al. Developing treatment guidelines for myasthenia gravis. Ann N Y Acad Sci 2018;1412:95-101.

10. Moslehi JJ, Salem JE, Sosman JA, et al. Increased reporting of fatal immune checkpoint inhibitor-associated myocarditis. Lancet 2018;391:933.

11. Möhn N, Beutel G, Gutzmer R, et al. Neurological immune related adverse events associated with nivolumab, ipilimumab, and pembrolizumab therapy-review of the literature and future outlook. J Clin Med 2019. doi: 10.3390/jcm8111777.

12. Makarious D, Horwood K, Coward JIG. Myasthenia gravis: an emerging toxicity of immune checkpoint inhibitors. Eur J Cancer 2017;82:128-36.

13. Fazel M, Jedlowski PM. Severe myositis, myocarditis, and myasthenia gravis with elevated anti-striated muscle antibody following single dose of ipilimumab-nivolumab therapy in a patient with metastatic melanoma. Case Reports Immunol 2019;2019:2539493.

14. Werner JM, Schweinsberg V, Schroeter M, et al. Successful treatment of myasthenia gravis following PD-1/ CTLA-4 combination checkpoint blockade in a patient with metastatic melanoma. Front Oncol 2019;9:84.

15. Johansen A, Christensen SJ, Scheie D, et al. Neuromuscular adverse events associated with anti-PD-1 monoclonal antibodies: systematic review. Neurology 2019;92:663-74.

16. Yuen C, Fleming G, Meyers M, et al. Myasthenia gravis induced by avelumab. Immunotherapy 2019;11:1181-5.

17. Johnson DB, Balko JM, Compton ML, et al. Fulminant myocarditis with combination immune checkpoint blockade. N Engl J Med 2016;375:1749-55.

18. Kimura T, Fukushima S, Miyashita A, et al. Myasthenic crisis and polymyositis induced by one dose of nivolumab. Cancer Sci 2016;107:1055-8.

19. Ji C, Roy MD, Golas J, et al. Myocarditis in cynomolgus monkeys following treatment with immune checkpoint inhibitors. Clin Cancer Res 2019;25:4735-48.

20. Anquetil C, Salem JE, Lebrun-Vignes B, et al. Immune checkpoint inhibitor-associated myositis. Circulation 2018;138:743-5.

21. Kao JC, Brickshawana A, Liewluck T. Neuromuscular complications of programmed cell death-1 (PD-1) inhibitors. Curr Neurol Neurosci Rep 2018;18:63.

22. Kolb NA, Trevino CR, Waheed W, et al. Neuromuscular complications of immune checkpoint inhibitor therapy. Muscle Nerve 2018. [Epub ahead of print].

23. Thompson JA, Schneider BJ, Brahmer J, et al. Management of immunotherapy-related toxicities, version 1.2019. J Natl Compr Canc Netw 2019;17:255-89.

24. Schwartz J. Evidence-based guideline update: plasmapheresis in neurologic disorders. Neurology 2011;77:e105-6; author reply e106. 
25. Dhawan PS, Goodman BP, Harper CM, et al. IVIG versus PLEX in the treatment of worsening myasthenia gravis: what is the evidence?: a critically appraised topic. Neurologist 2015;19:145-8.

26. Bomze D, Hasan Ali O, Bate A, et al. Association between immune-related adverse events during anti-PD-1 therapy and tumor mutational burden. JAMA Oncol 2019. [Epub ahead of print].

27. Mahmood SS, Fradley MG, Cohen JV, et al. Myocarditis in patients treated with immune checkpoint inhibitors. J Am Coll Cardiol 2018;71:1755-64.

28. Esfahani K, Buhlaiga N, Thébault P, et al. Alemtuzumab for immune-related myocarditis due to PD-1 therapy. N Engl J Med 2019;380:2375-6.
Cite this article as: Xing Q, Zhang ZW, Lin QH, Shen LH, Wang PM, Zhang S, Fan M, Zhu B. Myositis-myasthenia gravis overlap syndrome complicated with myasthenia crisis and myocarditis associated with anti-programmed cell death-1 (sintilimab) therapy for lung adenocarcinoma. Ann Transl Med 2020;8(5):250. doi: 10.21037/atm.2020.01.79 